\title{
Performance Analysis of OFDM System Augmented with Diverse Combination of Companding Techniques and Diverse Transforms
}

\author{
Shiwani Sandal and Lavish Kansal \\ School of Electronics and Electrical Engineering, \\ Lovely Professional University, Punjab, India \\ shiwanisandal@gmail.com,lavish.15911@lpu.co.in
}

\begin{abstract}
This paper proposes a companding plan, where little signals are augmented and vast signs are diminished, to lessen the Peak-to-Average Power Ratio (PAPR). Simulation comes about demonstrate that the proposed strategy has a point of interest in any event when contrasted and the traditional strategies, i.e. improved PAPR. To reduce this PAPR we have many reduction techniques but most of the techniques have not considered both complexity as well as the main factor PAPR. Here a new combinative scheme is proposed which combines companding with unique transforms and precoding techniques to reduce the PAPR at the appropriate level. The comparative analysis for proposed techniques is shown for PAPR as well as for Bit error rate (BER).
\end{abstract}

Keywords: OFDM, PAPR, Commanding, Transforms

\section{Introduction}

An OFDM system has been utilized as a part of mobile communication system. The interest for high information rates has turned into an essential consider the most recent decade. Additionally, the following eras of correspondence system ought to give high bit rates. The old single bearer system can't deal with these requests. Multicarrier modulation system can accomplish the high information rates [1-2]. Prior to the unrest of computerized system, multicarrier system couldn't be executed with high exactness utilizing the analog segments. Presently, the conceivable execution of digital chips brings the likelihood of manufacturing multicarrier systems inside reach. The most imperative multicarrier system is the OFDM system, which can be created utilizing some IFFT chips. Despite the fact that OFDM is straightforward in usage, regardless it experiences a fundamental drawback. Accordingly, the output peak power is high contrasted and the normal power, and this issue is notable as the peak to-average power ratio (PAPR) [2]. High PAPR brings about intermodulation bending, which debilitates the multicarrier general execution. Diverse plans have been acquainted with consider the PAPR issue. The basic approach is by clipping the substantial amplitudes [3]. The PAPR can likewise be lessened by pre-coding [4]. Different plans have been recommended, for example, partial transmit sequences (PTS) [4-5], selected mapping (SLM) [6], and other methods [7]. However, clipping the magnitude is a non-linear operation; thus, out-of-band and in-band contortions will be watched. Along these lines, the bit error rate (BER) execution will debase. Nonetheless, in one of our past reviews, the BER corruption due to clipping was eliminated completely [8-9]. Coding is sufficient yet to the detriment of the bitrate. PTS and SLM are probabilistic methods; along these lines, no BER debasement will be seen, yet the cost is the boundless expansion in the computational disperses nature of the system. Hence, there is a trade-off between the BER and the computational intricacy.

Received (December 9, 2017), Review Result (February 6, 2018), Accepted (February 15, 2018) 


\section{Principle of OFDM}

The time-space representation of an OFDM image with $\mathrm{N}$ subcarriers can be represented as:

$$
x(t)=\frac{1}{\sqrt{N}} \sum_{k=0}^{N-1} X(k) e^{j 2 \pi f k t} \quad 0 \leq t \leq T s
$$

Ts is the OFDM symbols length, $f k=\frac{1}{T s}$

The high PAPR of an OFDM signal rises up out of the summation of the inverse discrete Fourier change (IDFT) handle as showed up in (1). The PAPR of an OFDM signal in the analog domain can be represented as:

$$
P A P R=\frac{\max 0 \leq t \leq T s\left(|x(t)|^{2}\right)}{E\left(|x(t)|^{2}\right)}
$$

E () denotes the expectation operator.

\section{Companding}

Here we are utilizing two companding systems: A-law companding and $\mu$-law companding.

\section{A-law companding:}

In the companding system, the compansion of OFDM signal occur at the transmitter and increase at the recipient [9]. In the A-law companding, the compressor trademark is piecewise, contained a straight segment for low level data sources and a logarithmic section for unpredictable state inputs. Figure 1. Displays the A-law compressor used as a piece of OFDM framework. The inside and out that truly matters utilized estimation of "A" is 87.6. The direct area of the trademark is for low level information sources however the logarithmic section is for unpredictable state inputs. It is numerically passed on as:

$$
S(X)= \begin{cases}\operatorname{Smax} \frac{A \frac{|x|}{x \max }}{(1+A)} \operatorname{Sgn}(x), & 0<\frac{|x|}{x \max } \leq \frac{1}{A} \\ \operatorname{Smax} \frac{\left[1+\log \left(A \frac{|x|}{x \max }\right)\right]}{1+\operatorname{logeA}} \operatorname{Sgn}(x), & \frac{1}{A}<\frac{|x|}{x \max } \leq 1\end{cases}
$$

Where $\mathrm{x}=$ input signal, $\mathrm{y}=$ output signal, $|x|=$ absolute value, Sgn is sign function having negative as well as positive values and $\mathrm{A}=87.6$ defined by CCITT.

\section{$\mu$-law companding:}

A $\mu$-law companding system uses $\mu$-law figuring companding change by compacting the pinnacle signal and extending the little signal for decreasing PAPR. An OFDM transmitter in mix of $\mu$-law/A -law companding is portrayed in Figure 1. 


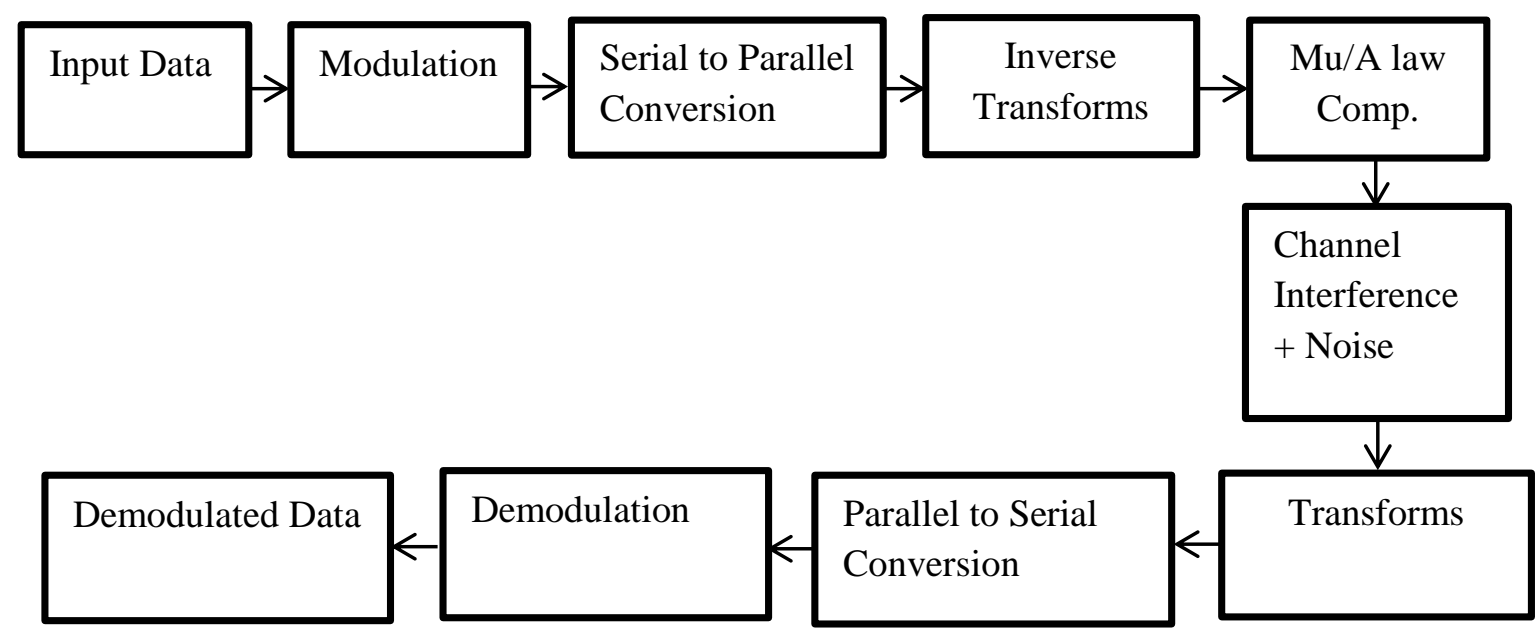

Figure 1. Block Diagram of $\mu$-law/A-law Companding in OFDM System

The basic equation used for $\mu$-law compression in this system is:

$$
S_{u}(t)=\frac{\ln \left[1+\mu \frac{|S(t)|}{\operatorname{Smax}(t)}\right]}{\ln (1+\mu)} \cdot \operatorname{Smax}(t) \cdot \operatorname{Sgn}(S(t))
$$

Where Sgn is a sign function, $\mu$ is the $\mu$-law parameter and generally its value is taken as 255, $\mathrm{S}(\mathrm{t})$ is instantaneous amplitude and Smax is peak amplitude.

\section{Proposed Methodology}

\section{(i) Hybrid Companding with Transforms}

We are utilizing diverse changes with companding change to decrease the PAPR. The transform block is utilized as a part of place of IFFT (Inverse Fast Fourier Transform) and converse of the change is utilized at the beneficiary side. The transforms used in combination of companding techniques are:

\section{$>$ DCT (Discrete Cosine Transform)}

Progression data $\mathrm{f}(\mathrm{x})$ of OFDM is changed with DCT and we will get progression data $\mathrm{F}(\mathrm{u})$, which changes the estimations of the segments of progression data. In any case, the progression structure won't be changed [5]. In the meantime, many zero parts will appear in the changed progression. That suggests, the energy of the signal focuses on some subsubcarriers, and PAPR is generally impacted by them. For example, $N=8$ and $f(x)=[1-1$ -1 - 1 - 1 ] we will get $\mathrm{F}(\mathrm{u})$

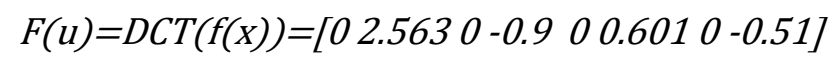

In equation (5), the biggest component of the succession subsequent to being changed is 2.563 , and alternate components are little or even 0 , which shows that the vitality of OFDM succession is engaged by DCT [6]. Besides, energy keeps the same after DCT, so the total energy stays unaltered.

$$
\left[\left(2.563^{\wedge} 2\right)+\left(-0.9^{\wedge} 2\right)+\left(0.601^{\wedge} 2\right)+\left(-0.51^{\wedge} 2\right)\right]=8
$$

So plainly DCT can center the signal energy around some little sub-carriers, which can lower down the PAPR. 


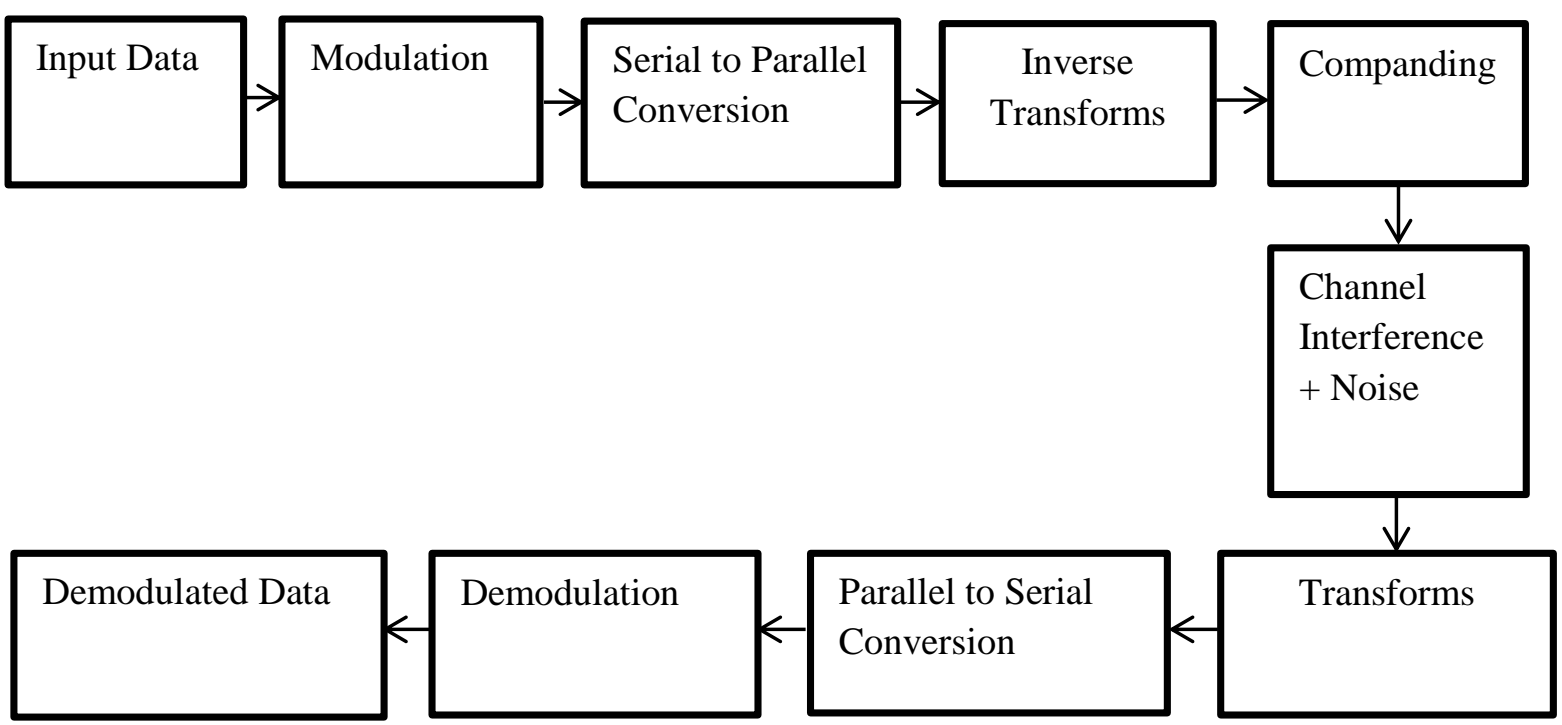

Figure 2. Hybrid Companding with Transform in OFDM System

\section{DST( Discrete Sine Transform)}

In arithmetic, the discrete sine change (DST) is a Fourier-related transform like the discrete Fourier transform (DFT), however using a totally real matrix [7-8]. It is equivalent to the whimsical parts of a DFT of by and large two fold the length, taking a shot at real data with odd symmetry (since the Fourier transform of a real and odd capacity is nonexistent and odd), where in a couple of varieties the data and also yield data are moved fundamentally a specimen[9]. A related change is the discrete cosine transform (DCT), which is equivalent to a DFT of real and even function.

$$
Y(k)=\sum_{n-1}^{N} x(n) \sin \pi \frac{k n}{N+1} k=1, \ldots \ldots \ldots . . . N
$$

$\mathrm{Y}$ is output signal; $\mathrm{x}$ is input signal $\mathrm{k}$ is $\mathrm{k}$ point DST.

\section{DWT( Discrete Wavelet Transform)}

The discrete wavelet change (DWT) is a straight change that works on an information vector whose length is integer power of two, changing it into a numerically distinctive vector of a similar length. It is an instrument that isolates information into various frequency parts, and after that reviews every segment with determination coordinated to its scale [10-11]. DWT is processed with a course of filtering's took after by a component 2 subsampling Figure 3.

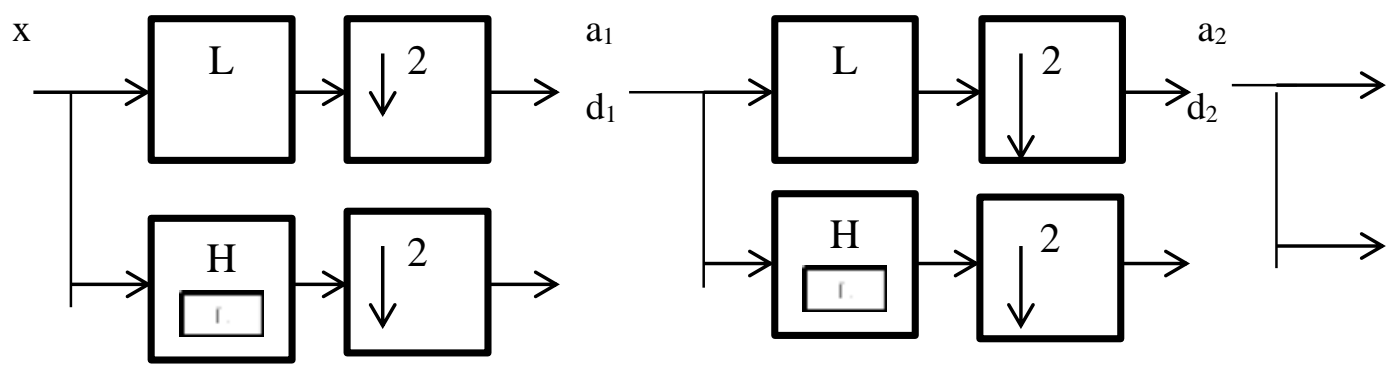

Figure 3. DWT Tree

$\mathrm{H}$ and $\mathrm{L}$ refers as high and low-pass filters, $\downarrow 2$ is subsampling. 


\section{(ii) Hybrid Companding with Precoding}

In precoding part we simply use above transforms in combination of IFFT block. The output of transform block is simply multiplied with IFFT matrix to get the improved results in PAPR. It is a pre -process system that is sent between the serial-to-parallel and the IFFT hinder at the transmitter side and same is done between parallel-to-serial and FFT obstruct at the recipient side yet opposite is taken of that transform which is utilized at the transmitter side [12].

$$
Y=P \otimes X
$$

$\mathrm{Y}$ represents the output vector, $\mathrm{P}$ is precoding matrix and $\mathrm{X}$ is input vector and $\otimes$ represents the element wise multiplication of precoding matrix and input vector.

Same set of transforms are used in this technique i.e., DCT, DST and DWT but with IFFT block. An OFDM transmitter joins precoding approach in frequency area with $\mu$-law companding plan in time space is appeared in Figure 4 [13-14].

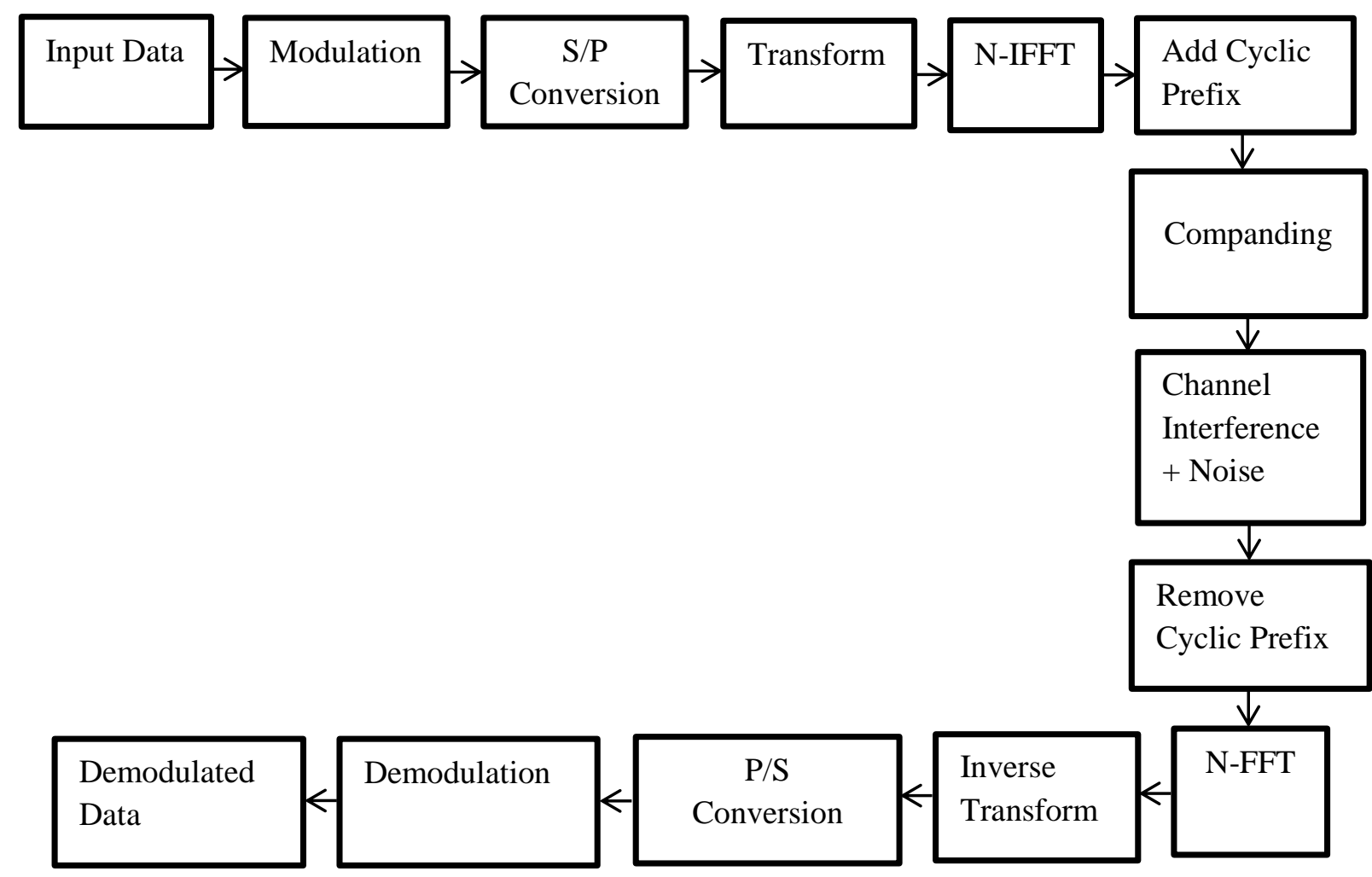

Figure 4. Hybrid Companding with Precoding Technique

\section{Performance Simulation}

This section shows the simulation of the proposed techniques which are explaining in above section and comparative analysis is done for PAPR and OFDM system. Firstly, analysis for conventional OFDM is done and further proposed techniques analysis are done with conventional OFDM system to know how much PAPR and BER gets reduced from the conventional OFDM system.

\section{(a) Analysis of OFDM system}

Simulations plot the complementary cumulative distribution function (CCDF), which is characterized as the likelihood that the PAPR surpasses a specific threshold PAPR0 for precoding, Companding method, Hybrid precoding and Hybrid transform companding 
procedure individually. Figure 5 (a) and (b) shows the analysis for conventional OFDM system and PAPR, for this is $11.2 \mathrm{~dB}$ and BER for this system is $42 \mathrm{~dB}$ approximately which is quite high. So to reduce these parameters various techniques are used to get the better noise immunity and spectral efficiency.

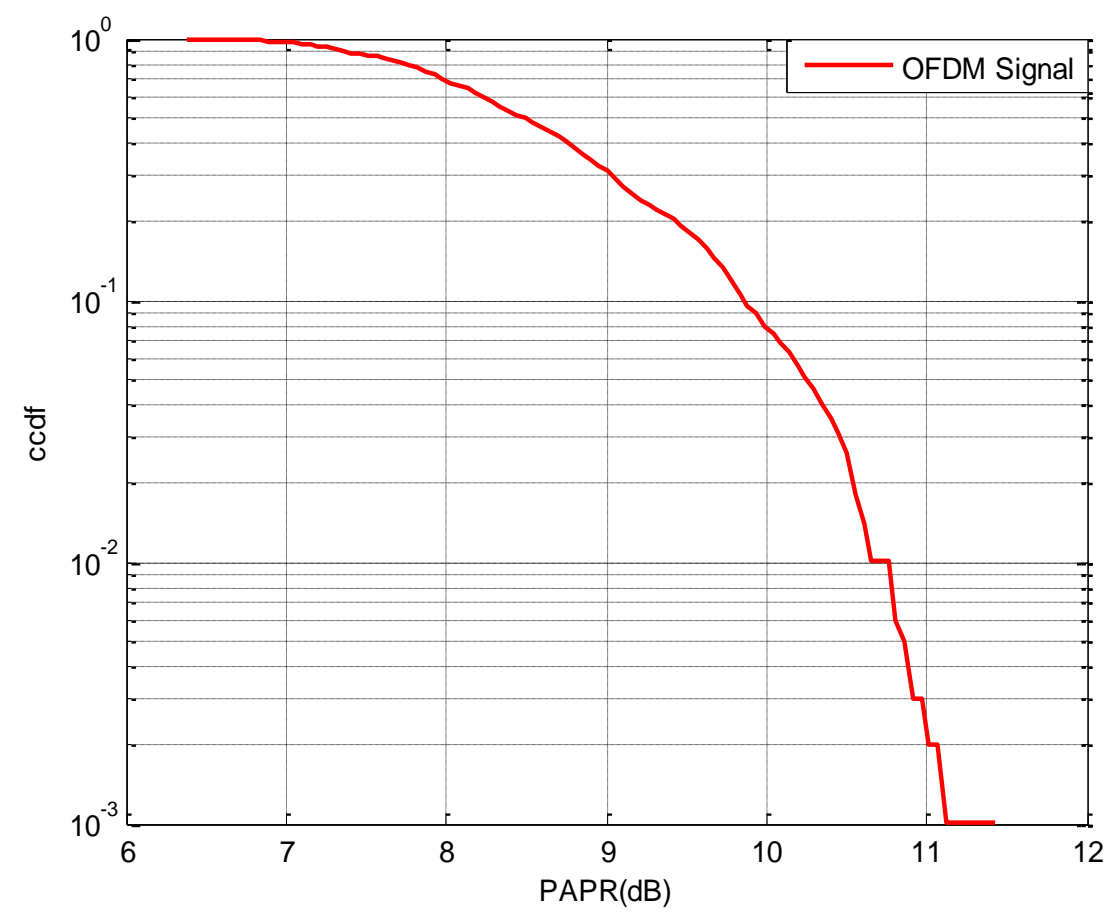

Figure 5(a). PAPR vs. CCDF of OFDM System

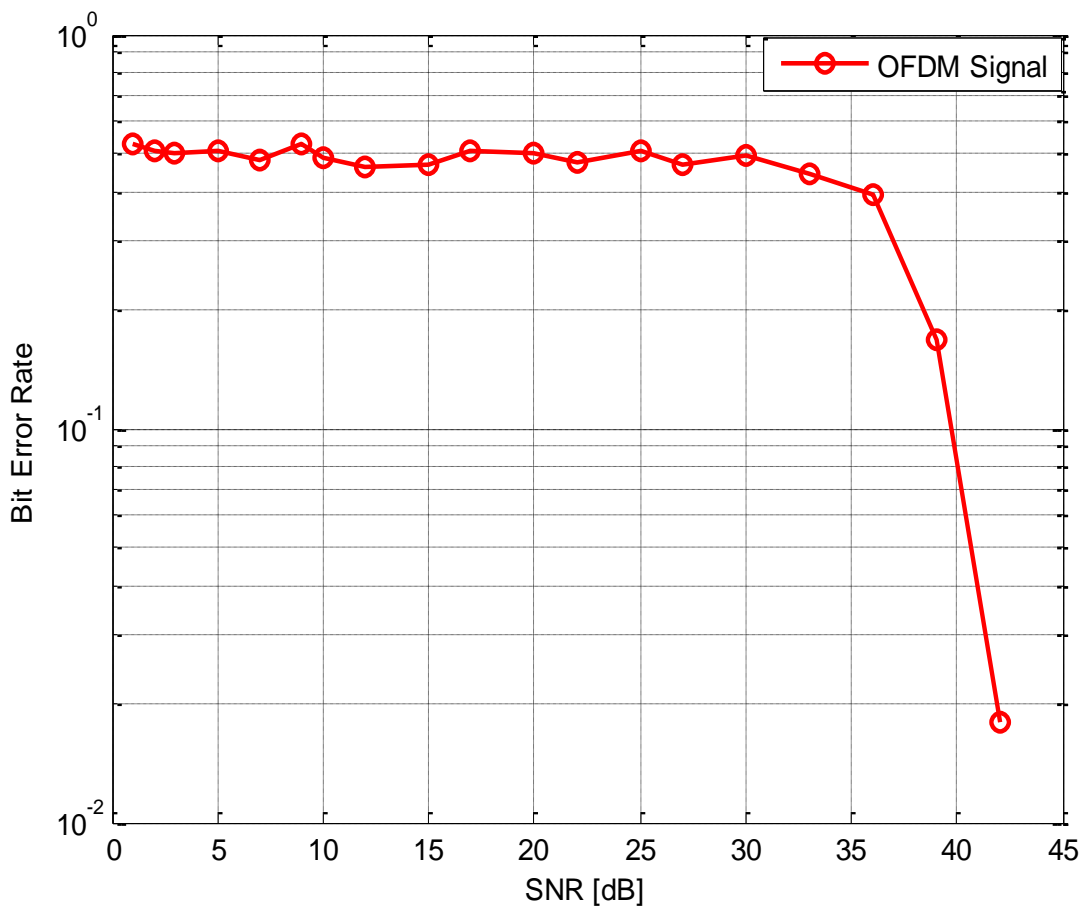

Figure 5(b). SNR vs. BER of OFDM System 


\section{(b) Analysis of OFDM with Hybrid Transform Companding}

With A-law Companding

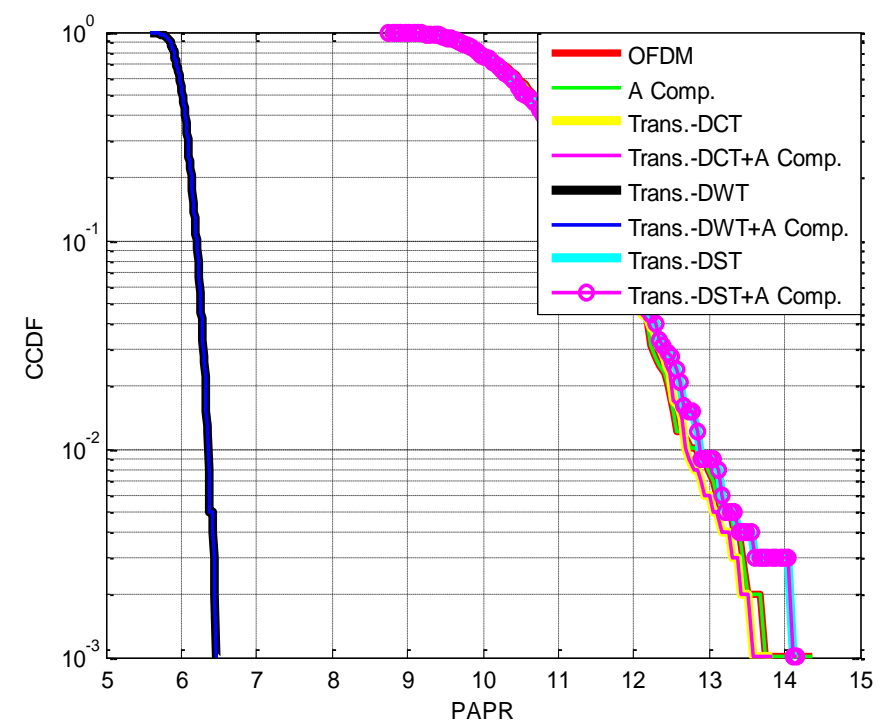

Figure 6(a). PAPR vs. CCDF of OFDM with Hybrid A-law Compnding.with Transforms

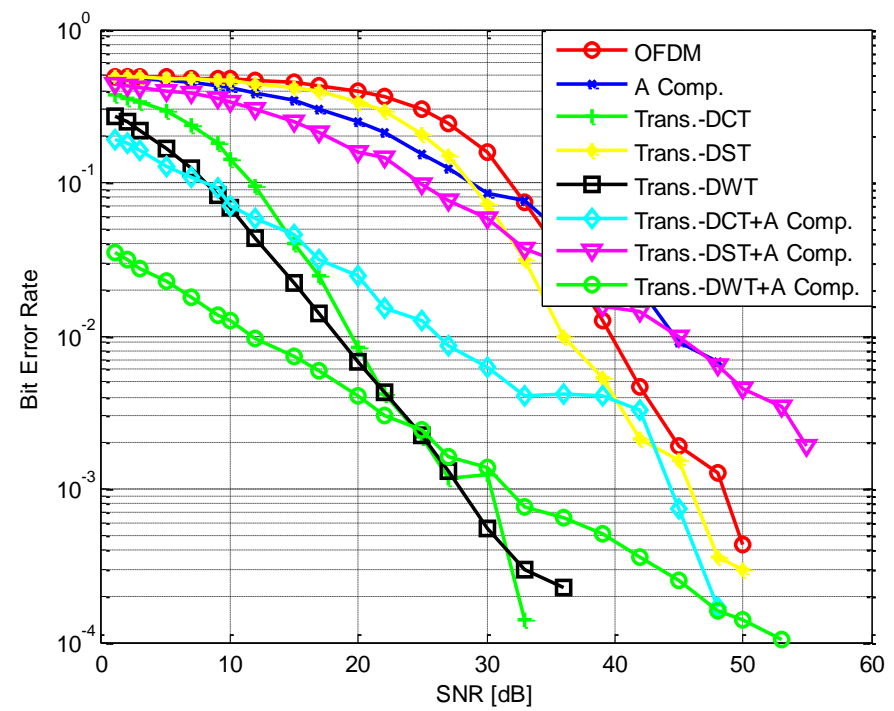

Figure 6(b). SNR vs. BER of OFDM with Hybrid A-law Companding with Transforms

In Figure 6(a) and (b) shows the PAPR and BER performance of various transforms with A-law Companding and compare with conventional OFDM. When A-law Companding is done on OFDM signal we get the PAPR approximately same as that of conventional OFDM signal i.e., 13.9dB approximately and BER for this signal 50dB approximately which is quite high. After applying the DCT, DST, DWT transform on the OFDM signal we get PAPR $13.5 \mathrm{~dB}, 14 \mathrm{~dB}, 6.5 \mathrm{~dB}$ respectively and BER for these transforms are $32 \mathrm{~dB}, 50 \mathrm{~dB}, 39 \mathrm{~dB}$ approximately. So with these BER is reduced as compared to conventional OFDM. Afterwards Hybrid technique which is proposed technique (Combination of A-law Companding with different transforms) is done on the 
companded signal having PAPR and BER for Hybrid DCT, DST, DWT with A-law Companding are $13.5 \mathrm{~dB}, 14 \mathrm{~dB}, 6.5 \mathrm{~dB}$ and $49 \mathrm{~dB}, 55 \mathrm{~dB}, 52 \mathrm{~dB}$ respectively. BER increases at the cost of reduced PAPR which is not good for the system.

With $\mu$-law Companding

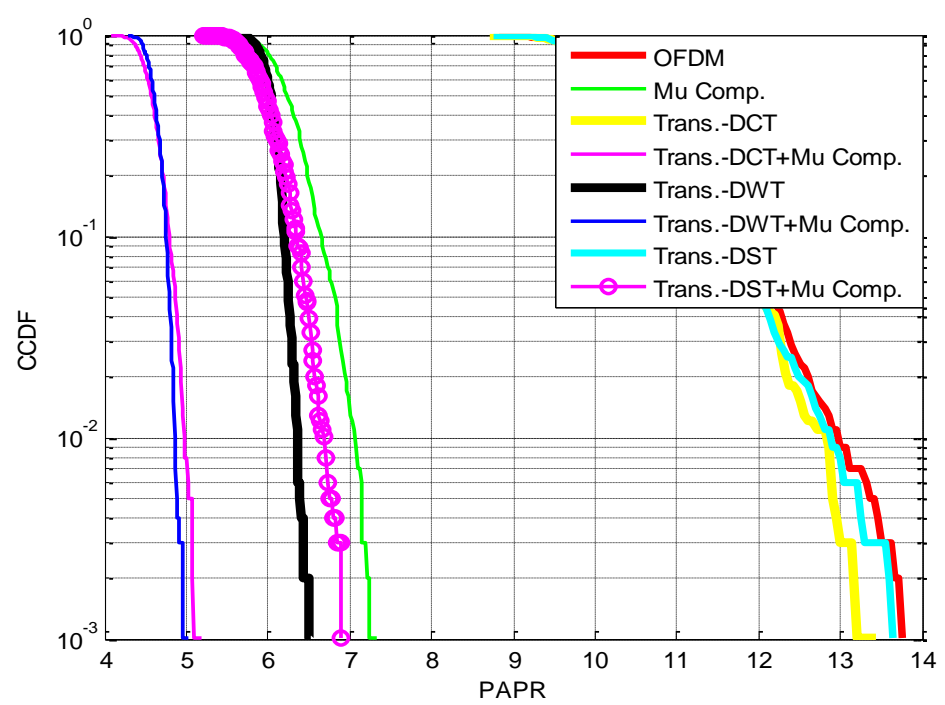

Figure 7(a). PAPR vs. CCDF of OFDM with Hybrid $\mu$-law Companding with Transforms

Same analysis is done for $\mu$-law companding. PAPR and BER for companded signal are $7.3 \mathrm{~dB}$ and $70 \mathrm{~dB}$ respectively. We get the good PAPR but with high value of BER which is not required. So proposed techniques are introduced. PAPR and BER for transforms DCT, DST, DWT are $13.1 \mathrm{~dB}, 13.5 \mathrm{~dB}, 6.5 \mathrm{~dB}$ and $70 \mathrm{~dB}, 50 \mathrm{~dB}$ and $39 \mathrm{~dB}$ approximately. Hybrid schemes of these DCT, DST, DWT transforms with A-law companding BER are $70 \mathrm{~dB}, 70 \mathrm{~dB}, 70 \mathrm{~dB}$ and PAPR are $5.1 \mathrm{~dB}, 6.9 \mathrm{~dB}, 4.9 \mathrm{~dB}$ approximately.

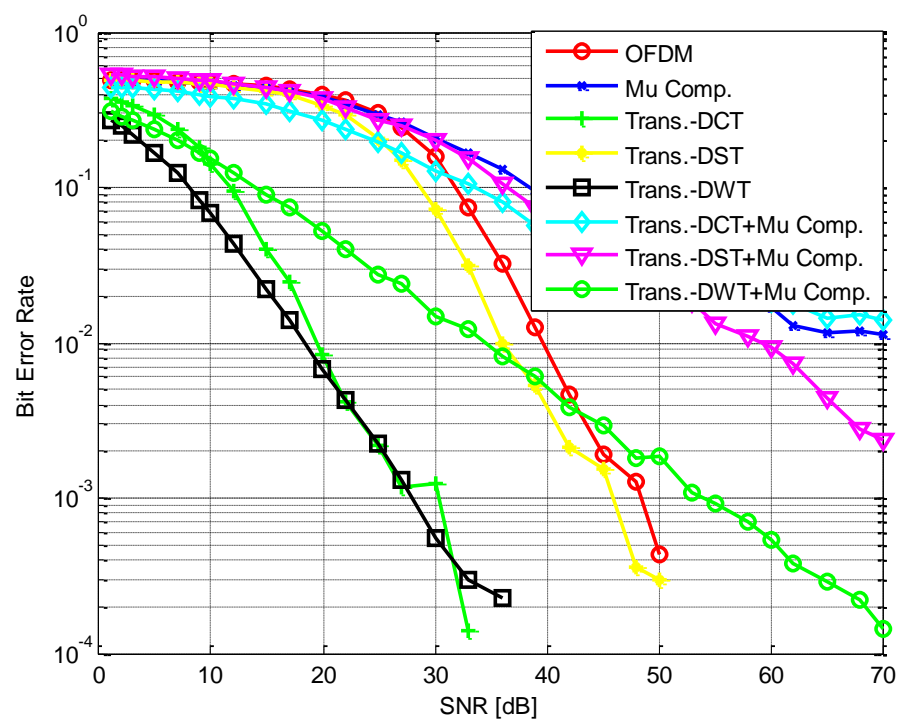

Figure 7(b). SNR vs. BER of OFDM with Hybrid $\mu$-law Companding with Transforms 
(c) Analysis of OFDM with Hybrid Precoding Companding

$>$ With A-law Companding

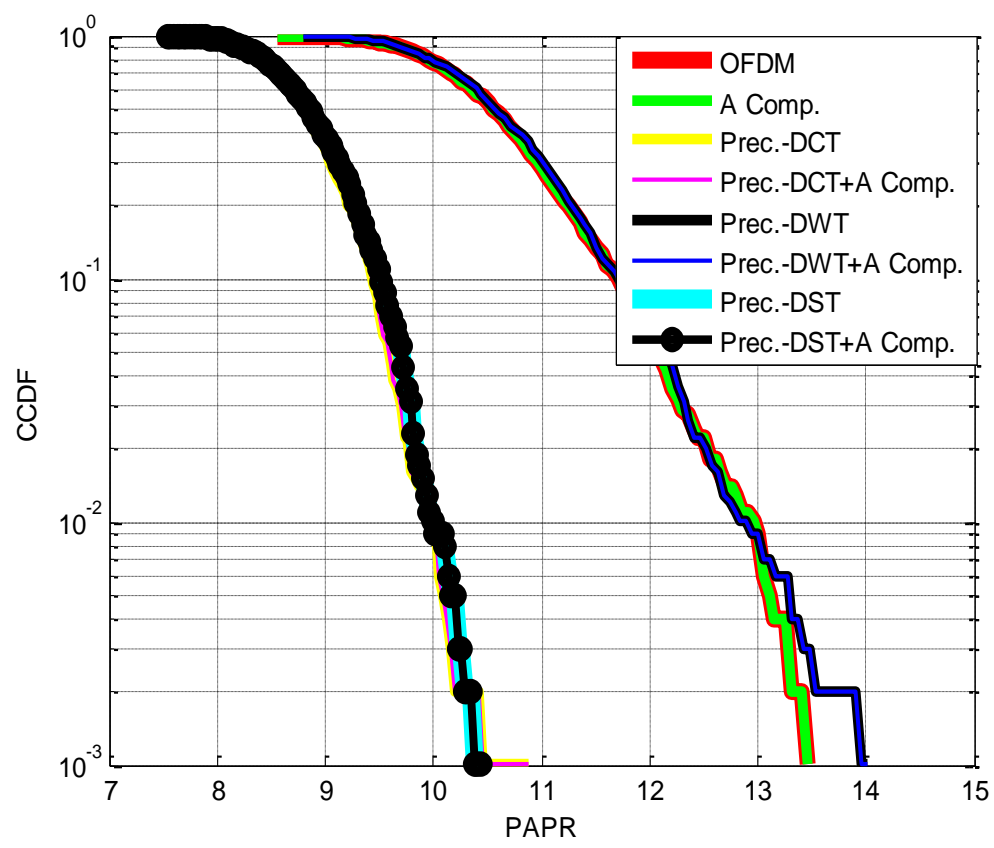

Figure 8(a). PAPR vs. CCDF of OFDM with Hybrid A-law Companding with Precoding Techniques

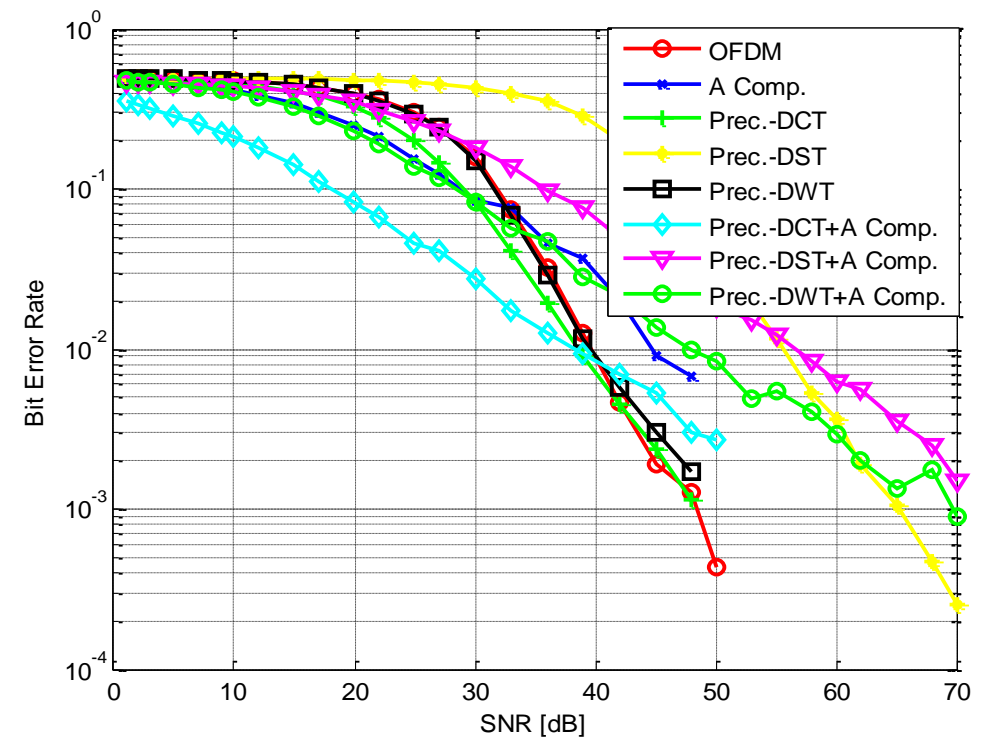

Figure 8(b). SNR vs. BER of OFDM with Hybrid A-law Companding with Precoding Techniques

Now we are evaluating the simulation for Hybrid Precoding schemes. PAPR for Precoded DCT, DST, DWT are $10.5 \mathrm{~dB}, 14 \mathrm{~dB}, 10.2 \mathrm{~dB}$ approximately and for Hybrid DCT, DST, DWT with A-law Companding PAPR are $10.3 \mathrm{~dB}, 14 \mathrm{~dB}, 10.3 \mathrm{~dB}$ respectively. Same simulation is done for BER. BER for Precoded DCT, DST, DWT are 50dB, 70dB, 
50dB respectively and for Hybrid DCT, DST, DWT with A-law Companding are 50dB, $70 \mathrm{~dB}$ and $70 \mathrm{~dB}$ approximately.

With $\mu$-law Companding

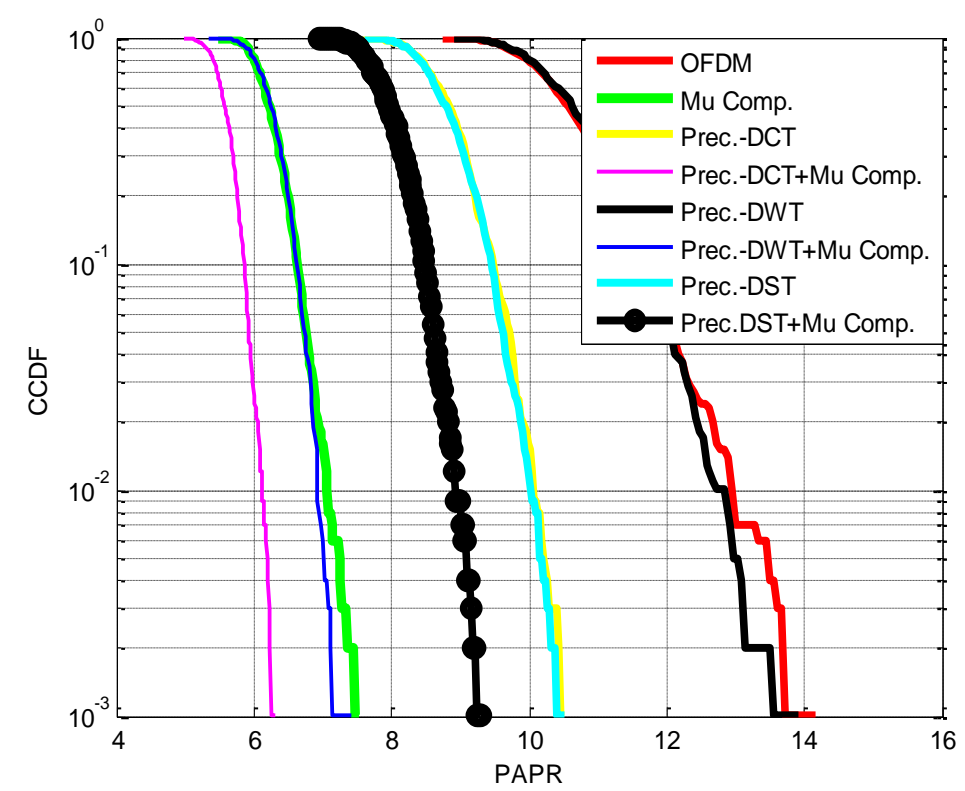

Figure 9(a). PAPR vs. CCDF of OFDM with Hybrid $\mu$-law Companding with Precoding Techniques

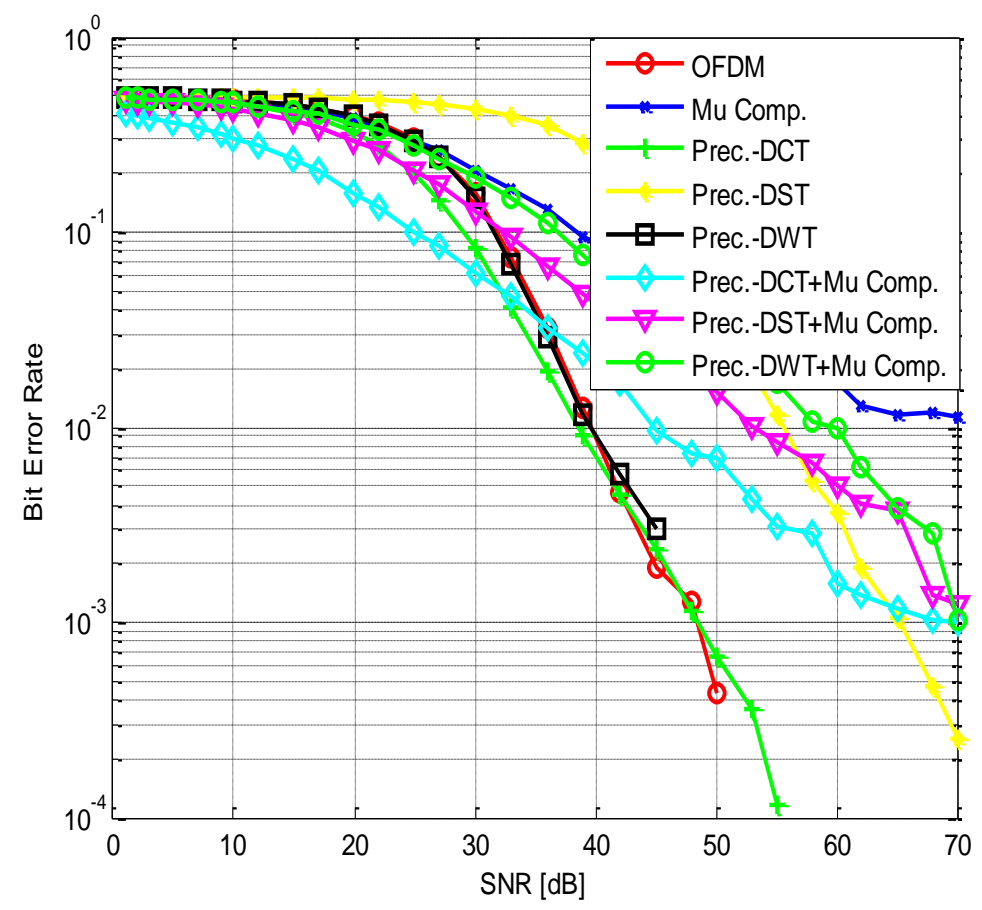

Figure 9(b). SNR vs. BER of OFDM with Hybrid $\mu$-law Companding with Precoding Techniques

Here we done the simulation for Hybrid Precoding schemes with $\mu$-law Companding. For Hybrid DCT, DST, DWT with $\mu$-law Companding PAPR are $6.2 \mathrm{~dB}, 8.8 \mathrm{~dB}, 6.5 \mathrm{~dB}$ 
respectively. Same simulation is done for BER. BER for Hybrid DCT, DST, DWT with $\mu$-law Companding are 70dB, 70dB and 70dB approximately.

\section{Conclusion}

The proposed approach which is the combining of transforms and precoding techniques with nonlinear companding techniques( A-law and $\mu$-law) has very significant results for PAPR reduction but for BER it is not counterbalance with PAPR. At one side we get very good reduction in PAPR but BER is gaining very high value. But this methodology reduced the complexity because in companding few parameters are scalable and also like SLM no side band information is required here. As compared to A-law companding we get the good results in $\mu$-law companding with the combination of DCT transform.

\section{References}

[1] S. H. Han \& J. H. Lee, "An overview of peak-to-average power ratio reduction techniques for multicarrier transmission", IEEE Personal Communications, vol. 12, no. 2, (2005), pp. 56-6.

[2] T. Jiang \& Y. Wu, "An Overview: Peak-to-Average Power Ratio Reduction Techniques for OFDM Signals", IEEE Transactions on Broadcasting, vol. 54, no. 2, (2008), pp. 257-268.

[3] R. W. Bauml, R. F. H. Fisher \& J. B. Huber, "Reducing the peak-to-average power ratio of multicarrier modulation by selected mapping", IEEE Electronics Letters, vol. 32, no. 22, (1996), pp. 2056-2057.

[4] S. B. Slimane, "Reducing the peak-to-average power ratio of OFDM signals through precoding", IEEE Transaction Vehicular Technology, vol. 56, no. 2, (2007), pp. 686-695.

[5] S. J. Heo, H. S. Noh, J. S. No \& D. J. Shin, "A modified SLM scheme with low complexity for PAPR reduction of OFDM systems", IEEE Transactions on Broadcasting, vol. 53, no. 4, (2007), pp. 804-808.

[6] C. Y. Hsu, H. C. Chao \& W. Y. Tsai, "PAPR Reduction of OFDM Signals Using Coefficient Array of Orthogonal Transform Kernels," IEEE 64th Vehicular Technology Conference, (2006), pp. 25-28.

[7] C. Y. Hsu \& H. C. Chao, "Novel SLM Scheme with Low Complexity for PAPR Reduction in OFDM System," IEICE Transactions on Fundamentals of Electronics, vol. E91-A, no. 7, (2008), pp.1689-1696.

[8] S. B. Slimane, "Reducing the Peak-to-Average Power Ratio of OFDM Signals through Precoding, "IEEE Transactions on Vehicular Technology, vol. 56, no. 2, (2007), pp. 686-695.

[9] X. Wang, T. T. Tjhung \& C. S. Ng, "Reduction of peak-to-average power ratio of OFDM system using a companding technique," IEEE Transaction on Broadcasting, vol. 45, no. 3, (1999), pp. 303-307.

[10] X. H. J. Lu, J. Zheng, J. Chuang \& J. Gu,"Reduction of peak-to-average power ratio of OFDM signals with Companding transform," IEEE Electronics Letters 12th, vol. 37, no. 8, (2001), pp. 506-507.

[11] T. Jiang \& G. Zhu, "Nonlinear Companding Transform for Reducing Peak-to-Average Power Ratio of OFDM Signals," IEEE Transactions on Broadcasting, vol. 50, no. 3, (2004), pp. 342-346.

[12] T. Jiang, Y. Yang \& Y. H. Song, "Exponential Companding Technique for PAPR Reduction in OFDM Systems," IEEE Transactions on Broadcasting, vol. 51, no. 2, (2005), pp. 244-248.

[13] Y. Jiang, "New companding Transform for PAPR Reduction in OFDM," IEEE Communications Letters, vol. 14, no. 4, (2010), pp. 282-284.

[14] I. M. Mahafeno, Y. Louet and J. F. Helard, "Peak-to-average power ration reduction using second order cone programming based tine reservation for terrestrial digital video broadcasting systems", IET Communications, vol. 3, no. 7, (2009), pp. 1250-1261. 
International Journal of Control and Automation

Vol. 11, No. 7 (2018) 